J. Astrophys. Astr. (xxxx) xx, 000-000

\title{
Core-collapse Supernovae and Gamma-ray Bursts in TMT Era
}

\author{
S. B. Pandey ${ }^{1 *}$ \\ (1) Aryabhatta Research Institute of observational-sciences, Manora peak, Nainital 263 129, India
}

Received xxx; accepted xxx

\begin{abstract}
.
Study of energetic cosmic explosions as a part of time domain astronomy is one of the key areas that could be pursued with upcoming Giant segmented optical-IR telescopes with a very large photon collecting area applying cutting edge technology. Existing 8-10m class telescopes have been helpful to improve our knowledge about Core-Collapse Supernovae, Gamma-ray Bursts and nature of their progenitors and explosion mechanisms. However, many aspects about these energetic cosmic explosions are still not well-understood and require much bigger telescopes and back-end instruments with high precision to address the evolution of massive stars and high-redshift Universe in more detail. In this presentation, possible thrust research areas towards CoreCollapse Supernovae and Gamma-ray Bursts with the Thirty Meter Telescope and back-end instruments are presented.
\end{abstract}

Key words: Massive stars, Core-collapse, Supernovae, Gammaray bursts, Optical, Telescopes

\section{Introduction}

The astronomical community with the aid of ground/space based telescopes has made tremendous progress over the last hundred years or so, in understanding many aspects of our observable universe. The findings include: discovery of exo-planetary systems, evidence for an accelerating universe, detailed identification and monitoring of the orbits of the asteroids and comets that may pose great dangers to the inhabitants of the Earth, and many more. Astronomers across the world feel a great need for new generation very large telescopes to probe the Universe much deeper, to unravel its

\footnotetext{
*e-mail: shashi@aries.res.in
} 
formation and evolution, and to discover the existence of life elsewhere in the Universe. With the current combination of $8-10 \mathrm{~m}$ class ground-based telescopes and the Hubble Space Telescope, study about Core-Collapse Supernovae (CCSNe) and Gamma-ray Bursts (GRBs) have been able to provide a great deal of information about the fate of evolution of massive stars $(>8-10 M \odot)$ and the underlying physical mechanisms (Woosley \& Bloom 2006; Langer 2012 and references therein). The unprecedented light gathering power and spatial resolution of the Thirty Meter Telescope ${ }^{1}$ (TMT) and the back-end instruments ${ }^{2}$ will allow us to study distant lighthouses like CCSNe and GRBs, could be studied in more detail probing the high-redshift Universe, dark energy contents and epoch of re-ionization.

\subsection{The TMT Project}

The TMT project is one of the most innovative among the proposed megascience projects in the next decade. The project is aimed to build the world's most advanced and capable ground-based optical, near-infrared, and midinfrared observatory. It will integrate the latest innovations in precision control, segmented mirror design, and adaptive optics (AO). At the heart of the telescope is the segmented mirror, made up of 492 individual segments of $1.45 \mathrm{~m}$. Precisely aligned, these segments will work as a single reflective surface of $30 \mathrm{~m}$ diameter. TMT will be located just below the summit of Mauna Kea on Hawaii Island, at an elevation of 4050 meters. The performance of a ground based telescope is adversely affected by the Earth's atmospheric seeing. The fundamental goal of any AO system is to improve the telescope performance from seeing limited, meaning the image quality is limited by the atmosphere above the telescope, toward diffraction limited, meaning images as sharp as those that could be obtained with the same diameter telescope located in space. Even in seeing limited mode, TMT offers an order of magnitude improvement over existing observatories, mostly due to its light gathering capacity. The AO capability enables TMT to resolve objects by a factor of 3 better than the current 10-m class telescopes and 12 times better than the Hubble Space Telescope. As first generation instruments for TMT, The Wide Field Optical Spectrometer (WFOS) will provide near-ultraviolet and optical $(0.3$ to $1.0 \mu \mathrm{m})$ imaging and spectroscopy over a more than 40 square arc-minute field-of-view. The concept for the TMT Wide-Field Optical Spectrometer is the Multi-Object Broadband Imaging Echellette (MOBIE) spectrometer. Narrow Field Infrared Adaptive Optics System (NFIRAOS) is the TMT's adaptive optics system for infrared instruments $(0.8-2.5 \mu \mathrm{m})$. Two of these science instruments will be delivered for use with the Multi Conjugate Adaptive Optics-Laser Guide star

\footnotetext{
${ }^{1}$ www.tmt.org/documents

${ }^{2}$ http://www.tmt.org/observatory/instruments
} 
(MCAO-LGS) system at first light: IRIS-TMT, a near-infrared instrument with parallel imaging and integral-field-spectroscopy support; and IRMS, an imaging, multi-slit near-infrared instrument (Crompton, Simard \& Silva 2008, TMT_documents1,2,3). The suit of above first generation instruments with TMT along with synergy with the $E-E L T^{3}$ and $J W S T^{4}$ would also be very relevant studying CCSNe and GRBs in great details.

\subsection{Indian Historical Prospects}

India has made several notable contributions towards optical-NIR astronomy during the latter half of the last century and had put in great efforts to set up world class observing facilities, which culminated in the indigenous building of the 2.3m Vainu Bappu Telescope (VBT) in 1987. The most recent astronomy facilities which have been set up in the country are, IIA's 2.0m Himalayan Chandra Telescope (2003) at Hanle, Ladakh and the 2.0m IUCAA Girawali telescope (2006) at Girawali, near Pune. There are two upcoming $4.0 \mathrm{~m}$ class optical-NIR astronomical facilities led by ARIES, Nainital: the $3.6 \mathrm{~m}$ optical-NIR facility (Devasthal Optical Telescope) which is expected to be commissioned in 2013, and the proposed $4.0 \mathrm{~m}$ International Liquid Mirror Telescope, both being set-up at Devasthal (Sagar et al. 2012). India will soon launch a dedicated astronomy satellite, ASTROSAT, with multi-wavelength capabilities in the UV and X-ray wavelengths. Indian astronomers have also contributed towards studying stellar explosions and early universe apart from other areas of observational astronomy. Using the observational capabilities in the country during last two decades, Indian astronomers have been able to study many SNe (e.g. Ashok B N et al. 1987; Pandey et al. 2003; Sahu et al. 2006; Roy et al. 2008; Brajesh et al. 2013) and afterglows of GRBs (e.g. Sagar et al. 1999; Bhattacharya 2003; Pandey et al. 2003, 2004; Resmi et al. 2013) in detail.

\subsection{India-TMT}

India's participation in international projects was envisaged in the Astronomy and Astrophysics 'Decadal Vision Document 2004' sponsored by the Indian Academy of Sciences and the Astronomical Society of India. In this context, international consortia for mega telescope projects approached astronomy institutes in the country for India's participation. The Indian astronomy community after due diligence and thorough deliberations, arrived at the conclusion that TMT presents the best options for India and participation in the project at a $10 \%$ level would be optimal.

The Department of Science and Technology (DST) reviewed the proposal

\footnotetext{
${ }^{3}$ http://www.eso.org/public/teles-instr/e-elt.html

${ }^{4}$ http://www.jwst.nasa.gov/
} 
submitted by Indian astronomers and approved the observer status for India in the TMT project in June 2010. Since then, India has been participating in all the policy decisions and development activities of the project. The Aryabhatta Research Institute for Observational Sciences (ARIES), Nainital; the Indian Institute of Astrophysics (IIA), Bangalore; and the Inter-University Center for Astronomy and Astrophysics (IUCAA), Pune; are the three main institutes spearheading the efforts. Options for other Indian Institutes and Universities are open to join the ongoing efforts by India-TMT. The activities of India-TMT ${ }^{5}$ will be coordinated by the India TMT Coordination Center (ITCC), jointly funded by the Department of Science and Technology and Department of Atomic Energy, Government of India.

\section{Core Collapse Supernovae}

It is commonly recognized that CCSNe represent the final stages of the life of massive stars $\left(M>8-10 \mathrm{M}_{\odot}\right)$ (Heger, Fryer \& Woosley 2003; Anderson \& James 2009). Generally, the fate of massive stars is governed by its mass, metallicity, rotation and magnetic field (Fryer 1999; Woosley \& Janka 2005). Massive stars show a wide variety in these fundamental parameters, causing diverse observational properties among various types of CCSNe. The presence of dominant $\mathrm{H}$ lines in the spectra of Type II SNe strongly suggests that their progenitors belong to massive stars which are still surrounded by significantly thick hydrogen envelope before the explosion (Filippenko 1997). For a Salpeter IMF with an upper cut-off at $100 \mathrm{M}_{\odot}$, half of all Type II SNe are produced by stars with masses between 8 and $13 \mathrm{M}_{\odot}$. This means that more than half of the stars producing SNe are poor sources of ionizing photons and of UV continuum photons, and that the bulk of the UV radiation, both in the Balmer and in the Lyman continuum is produced by much more massive stars. So, both the H-alpha flux and the UV flux are measurements of the very upper part of the IMF (about $>40 M_{\odot}$ ), representing stars with masses larger than $8 \mathrm{M}_{\odot}$ and are least understood. The analysis of archival images of HST have been able to detect some of the type II SNe progenitors as red and yellow super-giants (Yoon et al. 2012, Van dyk et al. 2012). On the contrary, $\mathrm{H}$ and He deficient features are commonly observed in the spectra of Type Ib/c SNe and are supposed to have luminous Wolf-Rayet stars as the possible progenitors. However, search for Type Ib/c SNe progenitors in the archival deep images has thus far been unsuccessful. The rate of corecollapse $\mathrm{SNe}$ (II, Ib/c) is a direct measurement of the death of stars more massive than $8 \mathrm{M}_{\odot}$, although it is still a matter of debate whether stars with mass above $40 \mathrm{M}_{\odot}$ produce a 'normal' SNe-II and Ib/c, or rather collapse forming a black hole with no explosion, i.e. a "collapsar" (e.g. Heger \&

\footnotetext{
${ }^{5}$ tmt.iiap.res.in
} 


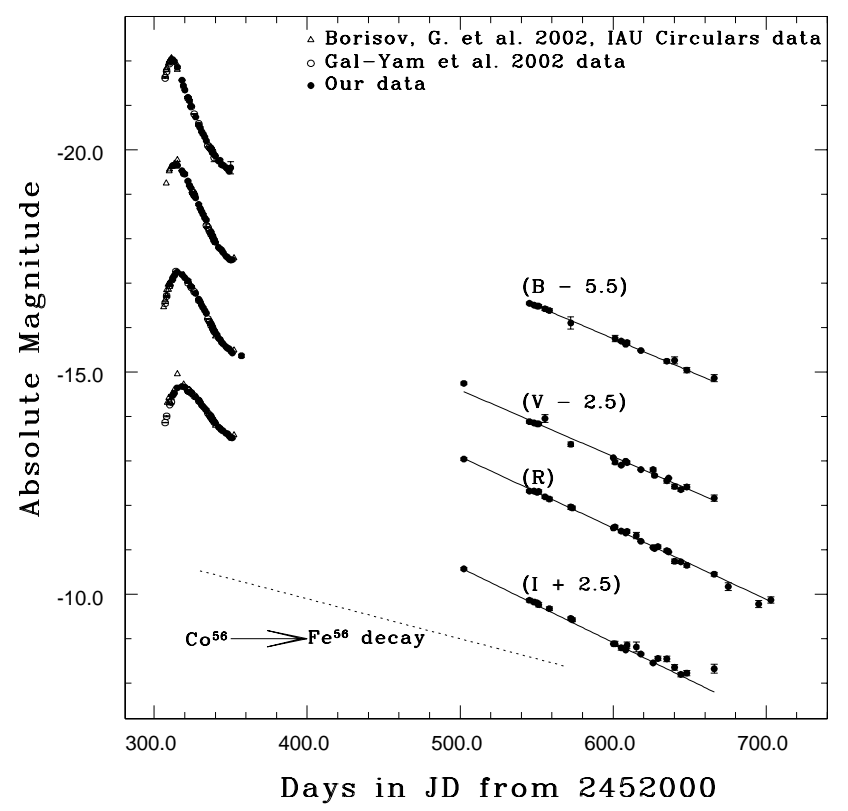

Figure 1. $B V R I$ light-curves of broad-lined Type Ic SN 2002ap discovered in M74 at z $\operatorname{sim} 0.002$, showing the typical decay nature of H-stripped CCSNe (Pandey 2006). SN 2002ap is more energetic than typical SNe of Type $\mathrm{Ib} / \mathrm{c}$ and exhibit close resemblance with SN 1998bw profile at later epochs.

Woosley 2002). These explosions and their possible progenitors are rather poorly understood research problems in astrophysics and a subject of great scientific interests (Taubenberger et al. 2009; Modjaz et al. 2011; Crowther 2013).

At low $(\mathrm{z}<0.1)$, intermediate $(0.1<\mathrm{z}<1.0)$ and high $(\mathrm{z}>1.0)$ redshifts many SN search programs such as CRST, PTF, KAIT, CfA, ES$S A N C E, S D S S, H S T-G O O D S$ are going on whereas many more are proposed to some in coming years e.g. SKYMAPPER, PANSTARRS, LSST, WFIRST, JWST. So far, the fraction of total number of discovered CCSNe $(\sim 76 \%)$ is found to be much more in comparison to the number of type Ia $\mathrm{SNe}(\sim 24 \%)$. Out of total number of discovered CCSNe, Type-II events are much more than Type Ib/c SNe (Lennarz, Altmann \& Wiebusch 2012). Statistically, the number of Type $\mathrm{Ib} / \mathrm{c}$ SNe in the local universe have been very less (only $4 \%$ of the total population) in comparison to number density of Type-II SNe and their possible progenitors are WR stars. No conclusive results have been found for the brightness distribution of CCNSe with their redshifts (Habergham, James \& Anderson 2012).

The TMT along with the back-end instruments is optimized both in OA 
and seeing-limited modes for the rapid response i.e. 10 minutes, extremely important for studies of time critical observations like SNe and GRBs. For example, IRIS-TMT spectra of a point source in one hour exposure time with $\mathrm{S} / \mathrm{N} \sim 10$ will be able to look at $\mathrm{J} \sim 24.1 \mathrm{mag}, \mathrm{H} \sim 23.7 \mathrm{mag}$ and $\mathrm{K} \sim$ 22.9 mag. Whereas seeing limited imaging of $\mathrm{S} / \mathrm{N} \sim 100$ will be bale to see sources upto $\mathrm{J} \sim 27.3 \mathrm{mag}, \mathrm{H} \sim 26.2 \mathrm{mag}$ and $\mathrm{K} \sim 25.5 \mathrm{mag}$ in a similar exposure time.

\subsection{CCSNe of Type II by TMT}

SNe IIn ( $3 \%$ of type-II populations) are the brightest SN type in the farUV and have long lived emission lines, makes them potential candidates to study in great detail at high redshifts. The average far-UV flux of Type IIn $\mathrm{SNe}$ at $\mathrm{z} \sim 2$ corresponds to $\mathrm{m}_{-} \mathrm{v} \sim 28 \mathrm{mag}$, enabled photometric detections in deep surveys. Moreover, the bright long-lived spectral features seen in case of Type-IIn SNe, remain above the threshold of 8-10m class telescope for years after the explosion, enabling WFOS-TMT kind of instrument to obtain rest-frame far-UV emission-line detections, spectral properties, and inferred kinematics of $\mathrm{z}>2$ Type IIn $\mathrm{SNe}$ and host galaxies. The rest-frame far-UV light would be red-shifted to optical wavelengths for $2<\mathrm{z}<6 \mathrm{SNe}$ and enables photometric detection in existing, and future, deep (m_v $\sim 27$ $28 \mathrm{mag}$ ) multi-epoch, wide-field optical surveys using 4-8m-class facilities. The ejecta of SNe IIn interact with cold circumstellar material expelled during previous evolutionary episodes and create extremely bright and longlived emission lines. Current 8-10m class telescopes do not have spectral sensitivity to detect Ly-alpha emission from $\mathrm{z}>3 \mathrm{SNe}$ of Type II. The strength and duration of the prominent emission lines permit spectroscopic detection of $2<\mathrm{z}<6$ using IRMS and WFOS-TMT for $\sim 3-15$ years after outburst (Cooke et al. 2009; Whalen et al. 2013a). Early time spectroscopy of Type IIn SNe during photospheric and shock-breakout phases using TMT is very important to understand the environments and the nature of the possible progenitors (Schawinski et al. 2008, Bufano et al. 2009). Late time spectral evolution of Type IIn SNe with TMT will be important to understand the geometry of the inner ejecta using evolution of line profiles and chemical enrichment of ISM and IGM.

On the other hand, CCSNe Type IIp are quite common in nearby universe and their atmosphere are dominated by $\mathrm{H}$ lines. Observational constraints on the possible progenitors of Type IIp SNe (Smartt 2009) using the pre-explosion images further make these locations as promising targets for TMT to put limits on the masses and types of the progenitors. It is also possible to probe Type IIp SNe at z $>1$ to study possible shock-breakout phase probing the underlying mechanisms of the possible progenitors of Type IIp SNe using TMT capabilities (Tominaga et al. 2011). Existing 8-10m class telescopes required 2-3 hours of exposure time to get a good $\mathrm{S} / \mathrm{N}$ nebular 
phase spectra $(\mathrm{z}<0.3)$ with Fe-II lines showing correlation with I-band luminosity, not seen in case of other Type II SNe. So, with the TMT capabilities, high-z SNe of Type IIp could be studied to be used as standard candles (Pozananski et al. 2012).

\subsection{CCSNe of Type $\mathrm{Ib} / \mathrm{c}$ by TMT}

Hydrogen-stripped Type Ib/c SNe (see Figure 1) are another potential targets for TMT to be studied in more details. The upcoming SN survey programs like LSST, WFIRST, JWST are expected to produce a sizable number of high-z Type Ib/c SNe. Massive Wolf-Rayet stars (> 25-30 $\left.\mathrm{M}_{\odot}\right)$ are supposed to be the potential progenitors for these SNe (Crowther 2007), so far not identified in per-discovery images taken by $H S T$ and ground-based 8-10m class telescopes (Maund, Smartt \& Schweizer 2005; Crockett et al. 2007, 2008). Another plausible way to produce Type Ib/c SNe is a lower mass helium-star in a binary channel with mass-loss mechanism (Podsiadlowski et al. 1992; Nomoto, Iwamoto \& Suzuki 1995). Majority of Type Ib/c SNe are found in star forming galaxies. Taking advantage of TMT OA and IFU capabilities, stellar population studies (Murphy et al. 2011; Anderson et al. 2012, Eldridge et al. 2013) close to the explosion site would tell more about the possible progenitors of Type Ib/c SNe.

Using WFOS-TMT capabilities, late time optical spectroscopy of type $\mathrm{Ib} / \mathrm{c}$ SNe could tell about kinematical, chemical information about the shocked ejecta and its interaction with the circumstellar material (Chevalier \& Fransson 2006). Late time optical spectroscopy could also help probing the mass loss history and evolutionary status of the progenitor stars (Leibundgut et al. 1991; Fesen et al. 1999). Time series optical spectroscopy of Type Ib/c SNe in nebular phases could also tell about the evolution of line widths and ratios of various line strengths, distinguishing the late time underlying physical mechanisms and ejecta structure. Recent observations of light echoes in supernova remnants have opened yet another method to determine the progenitors of supernovae. For example, light echoes from SN 1993J have been detected at the level of $22.3 \mathrm{mag} / \operatorname{arcsec}^{2}$ square by $H S T$ "V" band (F555W) observations. TMT class of telescopes will detect light echoes to V $28.5 \mathrm{mag} / \operatorname{arcsec}^{2}$ in galaxies well beyond the Virgo Cluster. With this capability one can determine the nature of SN types of all recorded explosions whose types (i.e. whether thermonuclear or core-collapse) are not known, in the past hundred years or so or of the SN remnants in the Local Group of Galaxies by faint object spectroscopy of the echoes of the light given out originally by the SN when it exploded (Milisavljevic et al. 2012). 


\subsection{Pair instability $\mathrm{SNe}$}

Pair instability supernovae (PISN) are thought to arise from extremely massive progenitors, possibly population-III stars above 100 solar mass (Rakavy \& Shaviv 1967, Barkat et al. 1967). Massive stars, with an initial mass range $140<\mathrm{M}<260 \mathrm{M}_{\odot}$ die in a thermonuclear runaway triggered by pair production instability (Kasen et al. 2011; Joggerst et al. 2012). Although PISN are rarely observed in the present, it is thought that they were very numerous in the distant past, among primordial, super-massive, low-metallicity rotating stars (Chatzopoulos \& Wheeler 2012), releasing huge energy of the order $10^{5} 3$ ergs and may synthesize considerable amount of $56 \mathrm{Ni}$ (upto $50 \mathrm{M}_{\odot}$ ). The PISN are characterized by peak magnitudes that are brighter than of Type II SNe and comparable, or brighter than type Ia, fall in category of super-luminous SNe of Type I and II observed in nearby universe (Quimby et al. 2013). Hydrogen lines are present in these SNe, arising from the outer envelope and have a long decay time $(\sim 1$ year $)$ due to large initial radii and large mass of material involved in the explosion. Very massive stars that can retain most of their mass against mass loss are those that with metallicity close to zero, and are hence expected at high redshifts. At redshift $\mathrm{z} \sim 2$, the PISN/SN ratio is expected to be 4-10 times higher than the observed local rate, for sub-solar mettalicity stars (Langer \& Norman 2006, Langer et al. 2007). Study of PISN using IRIS-TMT will also help in understanding the history of chemical enrichment, the nature of metal free stars at $\mathrm{z} \sim 6$ and the evolution of gaseous matter in the Universe (Scannapieco et al. 2005, Whalen et al. 2013b,c).

\subsection{Environment and Progenitors of CCSNe}

Host galaxies of CCSNe provide very useful information about the environments of these explosions, indirect clues about nature of their progenitors and evolution of high mass stars. With the help of ground-based $8-10 \mathrm{~m}$ class telescopes and HST imaging capabilities with a differential alignments of 10-130 mas, progenitors of some of the Type-II SNe have been identified, though limited to 10-120 Mpc only (Smartt et al. 2009; Modjaz et al. 2011). There are evidences, though not conclusive, for a correlation of masses of the progenitors with the types of CCSNe (Crowther 2012). However, estimates of host mettalicities does not show any such conclusive difference for various types of CCSNe (Boissier \& Prantzos 2009; Modjaz et al. 2011; Kelly and Krishner 2011). Locations of Type Ib/c SNe seems to be more centrally concentrated than Type II SNe in their host galaxies (Anderson \& James 2009; Arcavi et al. 2010).

With the help of AO capabilities of TMT and first generation instruments like WFOS and IRIS-IFU, mettalicies and star formation rate of the immediate environments of the host galaxies could be studied on the scales 
of kpc (Modjaz et al. 2008; Sahu et al. 2009; Anderson et al. 2010; Modjaz et al. 2011; Neill et al. 2011), providing more conclusive results about ages, mettalicity of the stellar population. TMT-IRIS and WFOS could be used to obtain rest-frame far-UV emission-line detections, spectral properties, and inferred kinematics of a large number of $\mathrm{z}>2$ galaxies hosting CCSNe of Type II and Ib/c, useful to understand the density, evolution, and dynamics of such events and the process of galaxy formation. There are evidences that some of the SNe Type IIn are PISN events (Langer 2012; Whalen et al. 2013a), providing a clue to understand the evolution of pop-III stars using follow-up observations of a larger sample of $z>2$ Type IIn SNe by LSST and other upcoming surveys.

\section{Gamma-ray Bursts}

GRBs are short lived ( $10^{-3}$ to $10^{3}$ seconds) extremely bright (Isotropic equivalent $\gamma$-ray energy $\sim 10^{50}-10^{55} \mathrm{erg}$ ) cosmological (redshift $\mathrm{z} \sim 0.01$ to 8.3) $\gamma$-ray sources, emitting photons of energy $\sim 10 \mathrm{keV}-90 \mathrm{GeV}$. They appear at random locations in the sky and have non-thermal spectrum (Piran 1999; Gehrels, Ramirez-Ruiz \& Fox 2009). Long-duration GRBs are now believed to be relativistic analogues of CCSNe explosions and are among the most energetic stellar-scale events known so far (Woosley \& Bloom 2006). The gamma-rays are not effected by dust absorption and optical extinction, hence could be seen at very high redshifts (Lamb \& Reichart 2000). Since last more than a decade, we have been able to understand GRBs and their properties using all possible ground and space-based observing facilities, however, many more questions about these enigmatic explosions are yet to be answered (Zhang 2007). GRBs are the only astrophysical sources known to be found in low-mettalicity environments and tools to probe the progenitors at high redshifts. Capabilities of TMT along with the first generation instruments specially the AO system with IRMS will be very helpful exploring high-redshift universe using GRBs.

\subsection{Afterglow light curves at late phases}

Followed by the prompt emission, ultra-relativistically ejected material interact with the surrounding medium through shocks and may produce afterglows, visible in all bands from $X$-ray to radio wavelengths. Afterglows

being longer-lasting than GRB prompt emission, provide a multi-band platform to study these energetic cosmic explosions in detail. The afterglow light curves generally show a power-law behavior. In general the flux $f_{\nu}$ from the afterglow follows a power law decay with time, a combination of several characteristic properties of the ejecta, represented as $f(\nu, t) \propto \nu^{\beta} t^{-\alpha}$ where $t$ is the time since the burst and $\alpha$ is the temporal flux decay index, $\nu$ is the frequency of observations and $\beta$ is the spectral index (Sari, Piran 
\& Narayan 1998, Wijers \& Galama 1999; Pandey et al. 2004). In case of most of the afterglows, generally the optical-IR emission is very faint ( $\mathrm{V}$ $\sim 23 \mathrm{mag}, 1-2$ day after the burst) and only 8-10m class telescopes could monitor the emission to observe the characteristic jet break time and other possible light curve features at late epochs (Sari, Piran \& Halpern 1999; ; Castro-Tirado et al. 1999; Mészáros 2002). Scaling similar bursts at z $\sim 1$ to $\mathrm{z} \sim 6-10$, the expected jet-break time and other light curve features (see Figure 2) would shift at later epochs and the afterglow emission would also shift towards IR bands due to Ly-alpha absorption, making the observations difficult even for existing 8-10m class telescopes. TMT imaging capabilities will be able to detect a good number of afterglows up to $\mathrm{z} \sim 6-10$ with the help of upcoming next generation GRB missions like EXIST.

One third of GRBs, terms as "dark GRBs", do not show any optical-IR emissions to a very deep limits at very early epochs even observed with 8$10 \mathrm{~m}$ class telescopes. The afterglow observations of these bursts at X-ray and $\mathrm{mm}$-wavelengths indicate that these bursts could either be extinguished by heavy dust of the host galaxies or exploded in a very low-density environments (Reichart \& Price 2002). It is also proposed that these bursts lie at very high redshifts (Lamb \& Reichart 2000) or these bursts are intrinsically very faint (Fynbo et al. 2001; Berger et al. 2002: Malendri et al. 2012: van der Horst et al. 2009: Jakobasson et al. 2004). Deep imaging capabilities of WFOS/IRIS-TMT with AO will be very useful to answer these questions about "dark GRBs".

\subsection{Time-resolved spectroscopy of afterglows}

Spectroscopy of afterglows of GRBs have many more useful implications than just measuring the distances. Early time afterglow spectra provide information about the absorption features due to fine structure transitions and resonance enabling accurate measurements about the dust content, chemical composition and complex gas kinematics of host and other intervening galaxies in the line of sight (Savaglio 2006, Prochaska et al. 2008). Present 8-10m class telescopes need lot of time to get spectra of GRB afterglows, for example, Subaru-FOCAS required $\sim 4$ hours for GRB 050904 ( $\mathrm{z}=6.295$ ), VLT-FORS2/ISAAC required $\sim 1.0$ hour for GRB $090423(\mathrm{z}=8.3)$ to get spectra just for the redshift determination (Totani et al. 2006; Tanvir et al. 2009). Gravitational collapse of rapidly evolving massive stars are supposed to produce long-duration GRBs, useful to probe young star forming regions

to quite far away distances (Tanvir et al. 2009). In fact, spectroscopy of optical-IR afterglows are proxies to probe the interstellar medium and intergalactic gas at cosmological distances in detail (Vreeswijk et al. 2007, 2013; D'Elia et al. 2009; Ledoux et al. 2009). Time resolved spectroscopy of optical-IR afterglows could also be used to understand the collisional excitation of fine structure lines, dust destruction effects due to UV/X- 
ray afterglows, measuring ISM densities and abundances (Vreeswijk et al. 2007). Taking advantage of rapid response modes along with the suite of back-end spectroscopic facilities (wavelength range $=0.31-0.62 \mu \mathrm{m} \& 2-2.4$ $\mu \mathrm{m} ; \mathrm{R}=1000-50000$ and 0.05 mas astrometry), TMT would be able to address many of these aspects in detail not possible to understand with help of current $8-10 \mathrm{~m}$ class telescopes. High resolution spectroscopy of GRBs with TMT-HRMS (at 0.34-1.0 $\mu \mathrm{m}, \mathrm{SN} / 100$, m_AB $\sim 20$ ) could be able to provide information about components effecting ISM upto kpc scales. Currently, the distances of absorbing gas from GRBs and study of fine structure lines have been done just in handful of cases only using VLT-MISTICI and X-SHOOTER instruments (D_Elia et al. 2007, 2009, 2011 ; Ledoux et al. 2009; Piranomonte et al. 2008; Vreeswijk et al. 2007, 2013).

\subsection{Observational cosmology and GRBs}

GRBs at high-z could be used as proxies to high-z quasars to investigate the epoch of re-ionization. As the end products of massive stars, GRBs briefly outshine any other source in the Universe and can be easily observed at cosmological distances. The recent discovery of a GRB at a redshift of $\mathrm{z} \sim$ 8.3 (Tanvir et al. 2009, Salvaterra et al. 2009), around similar redshifts of the most distant spectroscopically confirmed galaxies and quasars, establishes that massive stars were being produced and dying at these epochs. This recent detection of the distant GRBs using 4-10 m class telescopes clearly indicates that it is possible to detect and study many GRBs at $\mathrm{z}>10 \mathrm{using}$ the TMT and back-end instruments. GRBs being associated with individual stars, serve as signposts of star formation at the early epochs and can also provide a measure of the neutral fraction of IGM at the location of the burst. The observations of several GRBs at high-z would provide multiple lines of sight through the IGM and thus allow us to trace the process of re-ionization from its early stages.

Above properties suggest that GRBs could be good candidates to probe the cosmological models of the Universe with a much longer arm than Type Ia SNe (Soderberg et al. 2006, 2010). However, the huge dispersion (four orders of magnitude) of the isotropic GRB energy $\mathrm{E}_{i s o}$ makes them everything but standard candles barring the clustering of energy around $\mathrm{E}_{j e t} \sim 10^{51} \mathrm{erg}$ corrected for their jet opening angle (Frail et al. 2001) with a considerable scatter. The recent discovery of a very tight correlation between the collima-

tion corrected energy $\mathrm{E}_{j e t}$ and the GRB spectral peak energy $\mathrm{E}_{\text {peak }}$ are used as standard candles to constrain the cosmological parameters (Ghirlanda et al. 2004). The prospects for the use of GRBs as standard candles clearly depend on the increase of the number of detected GRBs which satisfy the $\mathrm{E}_{\text {peak }}-\mathrm{E}_{\text {jet }}$ correlation for a much longer redshift span. This will offer the unprecedented opportunity to investigate the nature of dark energy beyond what can be reached by study of Type Ia SNe. Cosmology with GRBs 


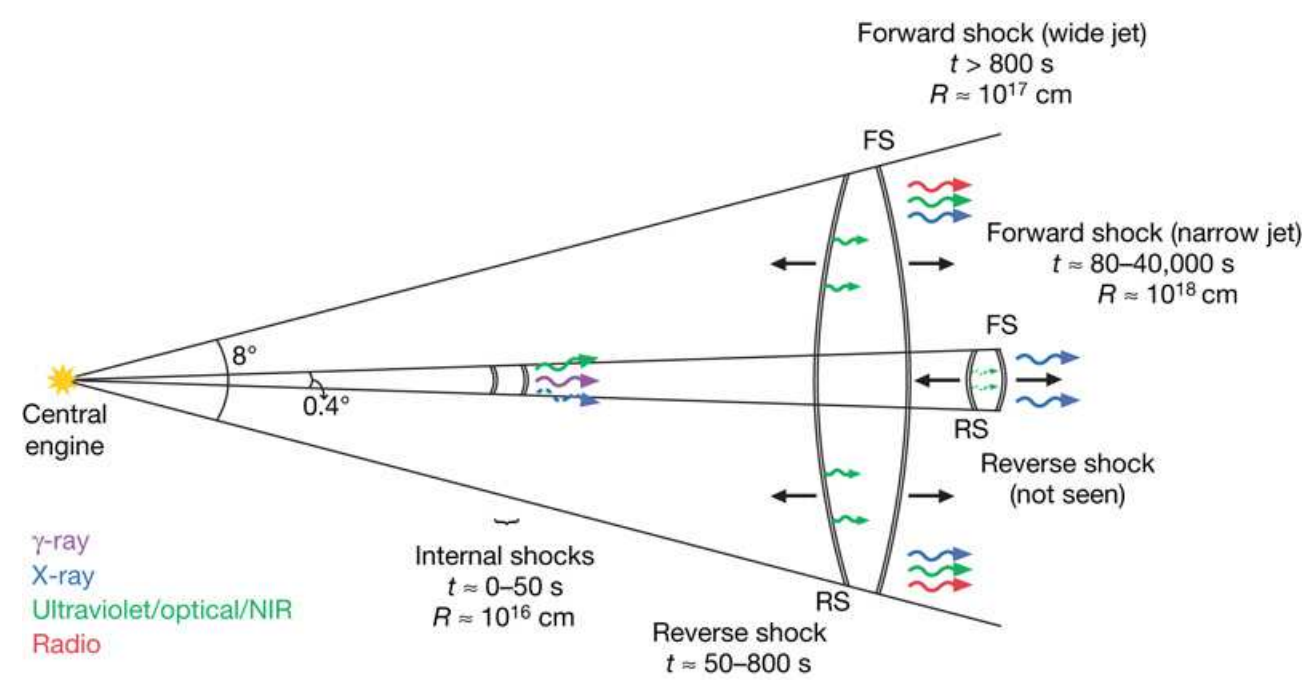

Figure 2. Schematic of the proposed two-component jet model of the afterglow of the "Naked-eye" GRB 080319B. The inner narrow-jet gives rise to the high energy emission whereas wider-jet is supposed to produce forward shock emission (Racusin et al. 2008). GRB 080319B ( $\mathrm{z} \sim 0.94)$ was very bright at optical frequencies and could have been observed even at redshift $\mathrm{z} \sim 16$ with the existing IR facilities hours after the burst.

through the $\mathrm{E}_{\text {peak }}-\mathrm{E}_{\text {jet }}$ ' correlation requires a set of observables, which are derived both from the GRB high energy emission (i.e. the prompt gammaray emission phase) and from the afterglow observations in the optical and IR band. In particular, the afterglow spectroscopic observation should provide the GRB redshift, while the long term (days to weeks) photometric monitoring of the afterglow emission allows measurement of the jet-break time. The latter allows us to estimate the GRB opening angle $\theta_{j e t}$ and, therefore, to derive the collimation corrected energy $\mathrm{E}_{j e t}$. TMT imaging capabilities at IR bands would be quite helpful constraining jet-break time in conjunction with facilities at other wavelength for very high redshift GRBs."

\subsection{Supernova connection of GRBs}

Some of the broad-lined Tpye Ib/c SNe are characterized by very high kinetic energies (Iwamoto et al. 1998; Nomoto et al 2004; Mazzali et al. 2008), have been observed to be associated both temporally and specially with long duration GRBs. Apart from spectroscopically confirmed such associations, several late time red-bumps have also been identified in the late time afterglow light-curves of long duration GRBs, close resemblance with SN 1998bw profile (See Figure 3 above; Cano et al. 2011). The GRBs associated Type $\mathrm{Ib} / \mathrm{c}$ SNe couple bulk of their energy to relativistic ejecta whereas ordinary 


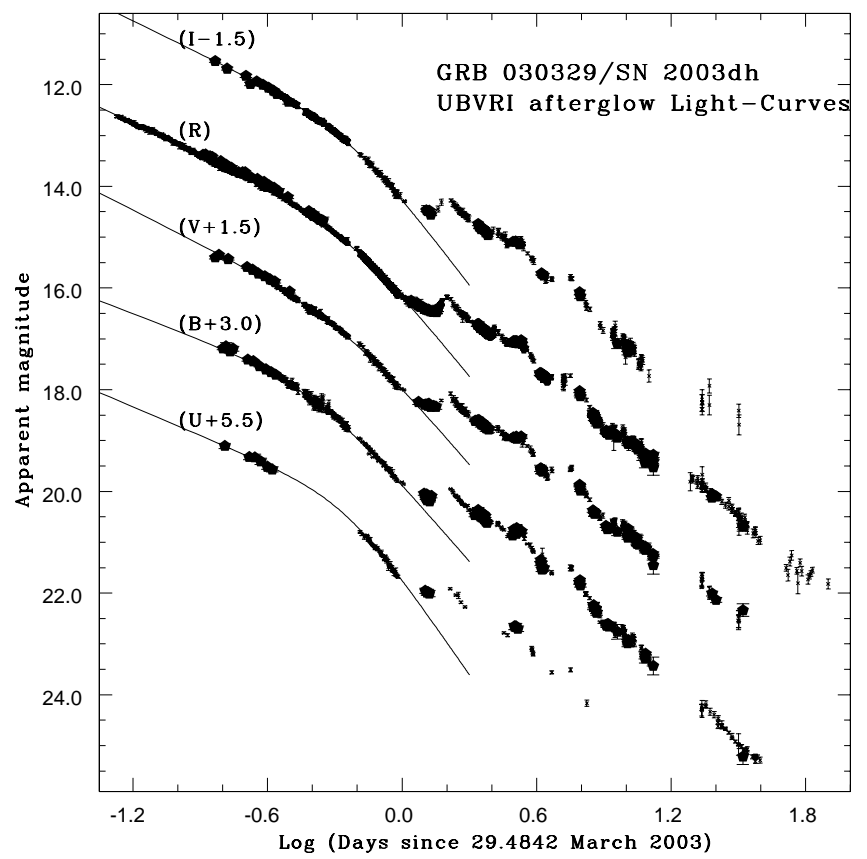

Figure 3. Light-curves of GRB 030329/SN 2003dh showing the deviation from the modeled light-curve of afterglows (Pandey 2006). The light-curve at late epochs are flattened due to contribution of the underlying SN 2003dh along with other light curve features. The time-resolved observations of such events could tell a lot about the explosion mechanism and underlying physical conditions.

$\mathrm{SNe} \mathrm{Ib} / \mathrm{c}$ couple less than a percent of their total energy to the fast material. Type Ib/c SNe related with GRBs have higher ejected mass in comparison to those not associated with GRBs (Mazzali et al. 2005,2006; Taubenberger et al. 2006; Valenti et al. 2008). Nature of the progenitors of SNe of Type $\mathrm{Ib} / \mathrm{c}$ is still not understood and that how do they differ from those associated with GRBs. However, the link to GRBs is a strong hint that GRBs associated Type Ib/c SNe could be significantly aspherical. If a jet is produced by a collapsing star (MacFadyen \& Woosely 1999), then only it can emerge and generate a GRB. A jet like explosion is required for GRBs from energetics considerations, indicating asphericity in the explosion mechanism. Ordinary Type Ib/c SNe are clearly distinguishable from the ones associated with the GRBs in terms of the relativistic ejecta produced by the central engine. There are some cases of low redshift Type Ib/c SNe with broad lines typical of hypernovae but not always associated with large luminosities typical of hypernovae (Mazalli et al. 2005; Valenti et al. 2008; Sahu et al. 2009; Drout et al. 2011). In the local universe, it has been seen that the rate of energetic broad-lined Ic SNe is similar to the rate of long-duration GRBs, which might 
indicate that most (or all) energetic Ic SNe produce GRBs (Podsiadlowski et al. 2004). However, the observed rate of production of WR stars (initial masses of $>40 \mathrm{M}_{\odot}$ ) in galaxies are much higher than the rate of broad-lined SNe of Type Ic, indicate that not all WR stars produce broad-lined Type $\mathrm{Ib} / \mathrm{c}$ SNe. Associations of Type Ib/c SNe have mostly been seen with lowluminosity nearby long duration GRBs (Hjorth \& Bloom 2011; Melandri et al. 2012; Sanders et al. 2012). Whether cosmological GRBs at higher redshifts are associated with SNe are quite challenging due to contamination by the afterglow and the host galaxy and is an open question to be addressed with TMT and other such facilities in near future.

\subsection{Host galaxies of GRBs}

GRBs being far away, lack direct identification of their progenitor stars. The evidence of what gives rise to GRBs therefore remains circumstantial. One important input to this body of evidence comes from the study of their host galaxies, and wherever possible, that of the immediate stellar neighborhood of the GRB. The GRB sample goes out to high redshift: the measured GRB redshifts at present have a median value of $\mathrm{z} \sim 2.7$. The host galaxies of GRBs that have been detected to date have their redshifts in the range 0.01-8.3. Clearly, a large fraction of the high redshift GRB host population remains to be discovered. The majority of the known hosts have magnitudes in the range $\mathrm{R} \sim 22-27 \mathrm{mag}$ and $\mathrm{K} \sim 20-25 \mathrm{mag}$, close to the limit of studies possible with the present-day 8-10m class telescopes (Savaglio et al. 2009). The TMT and the proposed back-end instruments will have an excellent opportunity to address questions exploring nature of GRB hosts. In addition to increasing the sample size at the high redshift end, TMT will enable detailed spectroscopic studies of the host galaxies. At present multi-band photometry of GRB hosts is being used to fit synthetic spectral models, from which the mass, metallicity, age and star formation rates are being deduced. The derived values suffer from very large uncertainties, making it difficult to reach definite conclusions about the nature of these galaxies and their population. TMT with the combination of OA and IFU will enable to study these objects spectroscopically, enhancing the reliability of model fitting and parameter extraction manifold. The present viewpoint that GRBs originate in sub-solar luminosity, actively star-forming galaxies with low metallicity can be seriously tested only by such detailed spectroscopic study about these host galaxies. Extending this study with TMT to a larger sample, especially at high redshifts would be able to tell more about the correlation of GRBs with low-metallicity progenitors. Morphological classification of GRB hosts, carried out mainly using the HST (Fruchter et al. 2006; Wainwright et al. 2007) so far, seems to suggest a preponderance of irregulars among them. The enhanced angular resolution of the TMTs compared to the present day ground based telescopes can be put to use in conducting such morphological 
classification of a larger sample of GRB hosts and their mass function. Multiband imaging with TMT could be used to understand the morphology of long and short duration host galaxies more in detail. This kind of study may also throw some light on whether the GRB production rate is influenced by galaxy mergers and the nature of galaxies hosting "dark GRB".

\section{Polarimetric studies of CCSNe and GRBs}

It is now established that afterglows of GRBs arise from synchrotron emission in a relativistically shocked ejecta, collimated outflow and emission is intrinsically polarized (Lazzati 2006; Wang \& Wheeler 2008). Evolution in the polarization as a function of viewing geometry as well as degree of orientation of the magnetic field (Medvedev \& Loab 1999) is expected in collimated outflows of GRBs. Models show that the maximum degree of polarization occurs around the time of geometric break called "jet-break" (Rossi et al. 2004). For GRB afterglows, optical imaging polarimetry have been successfully done in case of handful of GRBs and a polarization upto

9\% have been observed (Bersier et al. 2003, Wiersema et al. 2012a). For the observed afterglows in optical-NIR, lack of strong variability in the position angle of linear polarization indicate that magnetic field of the jet to be ordered rather than a random one. Even with the help of existing 8$10 \mathrm{~m}$ class telescopes like Keck and VLT, spectropolarimetric observations of GRB afterglows have been covered sparsely with no concluding results (Wiersema et al. 2012b). Some of the Type Ib/c SNe associated with GRBs have also shown polarization indicating towards asymmetry of the ejecta (photosphere/chemical inhomogeneity) for early photospheric phase of SNe (Kawabata et al. 2002, 2003; Tanaka et al. 2009). So far, spectropolarimetry of core-collapse SNe have only been possible using 8-10m class telescopes with rather low-resolutions (Hoffman et al. 2008) not providing any conclusive results about the structure of magnetic fields, rotational distortion of the progenitor stars and effect of interaction between circumstellar material and the ejected matter.

Bigger observing facilities like TMT will be able to collect many more photons, enabling to measure the polarization more accurately. However, the present design of the TMT with a three-mirror system is expected to produce considerable instrumental polarization due to tertiary mirror at Nasmyth focus (Tinbergen 2007) at optical-IR frequencies. To reduce the instrumental polarization some ideas have been suggested, like, putting a polarization modulator or an additional optical component in the optical path to cancel out the instrumental polarization. However, it is not easy to implement these possible solutions as the instrument will be rotating with the telescopes. To compensate the rotation of the telescope polarization, the possible ways are to put a achromatic half-wave plate or to bend the 
optical path using Fresnel Romb before the focal plane. It is highly required to optimize the design of TMT or other upcoming extremely large opticalIR telescopes to reduce the instrumental polarization to make best use of the collected photons from various faint celestial objects. Early phase spectropolarimetry of afterglows of GRBs and CCSNe will be able to tell us more about the explosion geometry, chemical structure, velocity, and composition of the SN ejecta.

\section{Conclusions}

The TMT and the back-end instruments are capable to address some of the un-answered questions about CCSNe and GRBs and their implications to understand the evolution of massive stars and star formation at high redshifts. Time resolved optical-IR spectroscopy of PISN and CCSNe of type II at $\mathrm{z}>2$ will be able to understand the shock-break out phase and interaction of ejecta with the circum-stellar matter in more details. Photometric identification of CCSNe at $\mathrm{z} \sim 2-6$ will be helpful to know about evolution of massive stars at high-z. Time resolved observations of of afterglows of GRBs would be quite helpful not only in understanding progenitors at high$\mathrm{z}$ and population of massive stars but also to prob the ISM and IGM in detail. Possible polarimetric observations using TMT would be able to tell about the geometry of the ejecta and structure of the magnetic field in these energetic cosmic explosions.

\section{Acknowledgments}

The author is thankful to organizers of TMT Science and Instrumentation workshop, 10-12 December 2012, Pune, India, to invite me to deliver the talk on SNe and GRBs. The author acknowledge the TMT documentation at www.tmt.org/documents/ while preparing this manuscript. The author is also thankful to Profs. Ram Sagar, D. Bhattacharya and G. C. Anupama to have a discussion on SNe and GRBs.

\section{References}

Anderson, J. P., Covarrubias, R. A., James, P. A. et al., 2010, MNRAS, 407, 2660

Anderson, J. P., Habergham, S. M., James, P. A. et al., 2012, MNRAS, 424, 1372

Anderson, J. P. \& James, P. A., 2009, MNRAS, 399, 559

Arcavi, I., Gal-Yam, A., Kasliwal, M. M. et al., 2010, ApJ, 721, 777

Ashok, B. N., Anupama, G. C., Prabhu, T. P. et al., 1987, JA\&A, 8, 195

Barkat, Z., Rakavy, G., \& Sack, N., 1967, Phys. Rev. Lett., 18, 379

Berger, E., Kulkarni, S. R., Bloom, J. S. et al., 2002, ApJ, 581, 981

Bersier, D., Stanek, K. Z., Winn, J. N., et al. 2003, ApJ, 584, L43

Bhattacharya, D., 2003, BASI, 29, 107 
Boissier, S. \& Prantzos, N., 2009, A\&SA, 503, 137

Bufano, F., Immler, S., Turatto, M., et al., 2009, ApJ, 700, 1456

Castro-Tirado, A. J., Zapatoras-Osorio, M. R., Caon, N. et al., 1999, Science, 283, 2069

Cano, Z., Bersier, D.; Guidorzi, C. et al., 2011, ApJ, 740, 41

Chatzopoulos, E. \& Wheeler, J. C., 2012, ApJ, 760, 154

Chevalier, R. A. \& Fransson, C., 2006, ApJ, 651, 381

Cooke, J., Coory, A., Chary, R. et al., 2009, White paper Astro2010 decadle survey, arXiv:0902:4602

Crockett, R. M., Eldredge, J. J., Smartt, S. J., et al., 2008, MNRAS, 391, L5

Crockett, R. M., Smartt, S. J., Eldredge, J. J., et al., 2007, MNRAS, 381, 835

Crompton, D., Simard, L. \& Silva, D., 2008, proceedings of the ESO Workshop "Science with the VLT in the ELT Era", arXiv:0801:3634

Crowther, P. A., 2007, ARA\&A, 45, 177

Crowther, P. A., 2013, submitted to MNRAS, arXiv:1210:1126

DÉlia, V., Campana, S., Covino, S., et al., 2011, MNRAS, 418, 680

DÉlia, V., Fiore, F., Meurs, E. J. A., 2007, A\&A, 467, 629

DÉlia, V., Fiore, F., Perna, R., et al., 2009, $A \& A$, 503, 437

Drout, M. R., Soderber, A. M., \& Gal-Yam, A. et al., 2011, ApJ, 741, 97

Eldridge, J. J., Fraser, M., Smartt, S. J., et al., 2013, submitted to MNRAS, arXiv:1301:1975

Fesen, R. A., Gerardy, C. L., Fillipenko, A. V. et al., 1999, AJ, 117, 725

Filippenko, A. V., 1997, ARA $\& A$, 35, 309

Frail, D. A., Kulkarni, S. R., Sari, R., 2001, ApJ, 562, L55

Fryer, C. L., 1999, ApJ, 522, 413

Fryer, C. L. \& Heger, A., 2005, ApJ, 623, 302

Fruchter, A. S., Levan, A. J., \& Strolger, L. et al. 2006, Nature, 441, 463,

Fynbo, J. P. U., Jensen, B. L., Gorosabel, J. et al., 2001, $A \mathscr{E} A$, 369, 373

Ghirlanda, G., Ghisellini, G., Lazzati, G., et al., 2004, ApJ, 613, L13

Gehrels, N., Ramirez-Ruiz, E., Fox D.B., 2009, ARA\&A, 47, 567

Habergham, S. M., James, P. A. \& Anderson, J. P., 2012, MNRAS, 424, 2841

Heger, A., Fryer, C. L. \& Woosley, S. E., 2003, ApJ, 591, 288

Heger, A \& Woosley, S. E., 2002, ApJ, 567, 532

Hjorth, J. \& Bloom, J., 2011, Chapter 9 in "Gamma-Ray Bursts", eds. C. Kouveliotou, R. A. M. J. Wijers, S. E. Woosley, Cambridge University Press, 2011 Journal-ref: Gamma-Ray Bursts, Cambridge Astrophysics Series 51, 169

Hoffman, J. L., Leonard, D. C., Chornock, R. et al., 2008, ApJ, 688, 1186

Iwamoto, K., Mazzali, P. A., Nomoto, K., 1998, Nature, 395, 672

Jakobasson, P., Hjorth, J., Fynbo, J. P. U. et al., 2004, ApJ, 617, L21

Joggerst, C. C. \& Whalen, D. J., 2011, ApJ, 728, 129

Kasen, D., Wooley, S. E., Heger, A., 2011, submitted to ApJ, arXiv:1101.3336

Kawabata, K. S., Jeffery, D. J.; Iye, M. et al., 2002, ApJ, 580, L39

Kawabata, K. S., Deng, J., Wang, L. et al., 2003, ApJ, 593, L19

Kelly, P. K. \& Krishner, R. P., 2012, accepted to ApJ, arXiv:1110.1377

Kumar, B., Pandey, S. B., Sahu, D. K., et al. 2013, MNRAS, 431, 308

Lamb, D. Q. \& Reichart, D. E., 2000, ApJ, 536, 1

Langer, N., 2012, ARAEAA, 50, 107 
Langer, N. \& Norman, C. A., 2006, ApJ, 638, L63

Langer, N., Norman, C. A., de Koter, A. et al., 2007, $A \mathscr{E} A$, 475, L19

Lazzati, D., 2006, NJPh, 8, 131

Lazzati, D., Covino, S., Alighieri, di S. et al., 2003, A $\mathscr{E} A$, 410, 823

Leibundgut, B., Krishner, R. P., Pinto, P. A. et al., 1991, ApJ, 372, 531

Lennarz, D., Altmann, D. \& Wiebusch, C., 2012, A\&SA, 538, 120

MacFadyen, A. I. \& Woosley, S. E., 1999, ApJ, 524, 262

Maund, J. R., Smartt, S. J. \& Schweizer, F., 2005, ApJ, 630, L33

Mazzali, P. A., Kawabata, K. S., Maeda, K. et al., 2005, Science, 308, 1284

Mazzali, P. A., Deng, J., Pian, E. et al., 2006, ApJ, 645, 1323

Mazzali, P. A., Valenti, S., Della Valle, M. et al. 2008, Science, 321, 1185

Medvedev, M. V. \& Loeb, A., 1999, ApJ, 526, 697

Melandri, A., Pian E., Ferrero P., et al. 2012, A\& $A$, 547, A82

Malendri, A., Sbarufatti, B., D'Avanzo, P. et al., 2012, MNRAS, 421, 1265

Mészáros, P., 2002, ARA $\& A, 40,137$

Milisavljevic, D., Fesen, R. A., Chevalier, R. A. et al., 2012, ApJ, 751, 25

Modjaz, M., Kewley, L., Bloom, J. S. et al., 2011, ApJ, 731, L4

Modjaz, M. Kewley, L., Krishner, R. P. et al., 2008, AJ, 135, 1136

Murphy, J. W., Jennings, Z. G., Williams, B. el al., 2011, ApJ, 742, 4

Neill, J. D., et al. 2011, ApJ, 727, 15

Nomoto, K. I., Iwamoto, K. \& Suzuki, T., 1995, PhR, 256, 173

Pandey, S. B., 2006, PhD Thesis

Pandey, S. B., Anupama, G. C., Sagar, R. et al. 2003, MNRAS, 340, 375

Pandey, S. B., Sahu, D. K., Resmi, L. et al., 2003, BASI, 31, 19

Pandey, S. B., Sagar, R., Anupama, G. C. et al., 2004, A\&A A, 417, 919

Podsiadlowski, Ph, Joss, P. C., \& Hsu, J. J. L., 1992, ApJ, 391, 246

Podsiadlowski, Ph, Mazzali, P. A., \& Namoto, K. et al., 2004, ApJ, 607, L17

Poznanski, D., 2013, arXiv:1304:4967

Poznanski, D., Nugent, P. E., Filippenko, A. V., 2008, accepted to ApJ, arXiv:1008:0877

Piran, T., 1999, Physics Reports, 314, 575

Piranomonte, S., Ward, P. A., Fiore, F., et al., 2008, A\&A, 492, 775

Prochaska, J. X., Sheffer, Y., Perley, D. A. et al., 2009, ApJ, 691, L27

Quimby, R. M., Yuan, F., Akerlof, C. et al., 2013, accepted to MNRAS, arXiv:1302:0911

Racusin, J. L., Karpov, S. V., Sokolowski, M. et al., 2008, Nature, 455, 183

Rakavy, G. \& Shaviv, G., 1967, ApJ, 148, 803

Resmi, L., Misra, K., Jhannesson, G. et al., 2013, MNRAS, 427, 288

Reichart, D. E. \& Price, P. A., 2002, ApJ, 565, 174

Rossi, E. M., Lazzati, D., Salmonson, J. D. et al., 2004, ApJ, 354, 86

Roy, R., Kumar, B., Benetti, S. et al., 2011, ApJ, 736, 76

Sagar, R., Kumar, B., Omar A., et al., 2012, SPIE, 8444, 1TS

Sagar, R., Pandey, A. K., Mohan, V., et al. 1999, BASI, 27, 3

Sahu, D. K., Anupama, G. C., Srividya, S. et al. 2006, MNRAS, 372, 1315

Sahu, D. K., Tanaka, M., Anupama, G. C. et al., 2009, ApJ, 697, 676

Salvaterra, R., Della Valle, M., Campana, S., et al., 2009, Nature, 461, 1258

Sanders, N. E., Soderberg, A. M., Levesque, E. M., et al., 2012, ApJ, 758, 132

Savaglio, S., 2006, New Journal of Physics, 8, 9, 195 
Savaglio, S., Glazebrook K., Le Borgne D., 2009, ApJ, 691, 182

Sari, R., Piran, T., Narayan, R., 1998, ApJ, 497, L17

Scannapieco, E., Madau, P., Woosely, S. et al., 2005, ApJ, 633, 1031

Schawinski, K. Stephen, J., Christian, W. et al., 2008, Science, 321, 223

Smartt, S. J., 2009, ARA\&A, 47, 63

Smartt, S. J., Eldridge, J. J., Crockett, R. M., et al., 2009,MNRAS, 395, 1409

Soderberg, A. M., Chakraborti, S., Pignata, G., 2010, Nature, 463, 513

Soderberg, A. M., Kulkarni, S. R., Price, P. A. et al., 2006, ApJ, 636, 391

Steele, I. A., Mundell, C. G., Smith, R. J. et al., 2009, Nature, 462, 767

Tanaka, M., Moriya, T. J., Yoshida H., et al., 2012, MNRAS, arxiv:1202:3610

Tanvir, N. R., Fox, D. B., Levan, A. J., et al., 2009, Nature, 461, 1254

Taubenberger, S., Valenti, S., Benetti, S., et al., 2009, MNRAS, 397, 677

Taubenberger, S., Pastorello, A., Mazzali, P. A. et al., 2006, MNRAS, 371, 1459

Tinbergen, J., 2007, PASP, 117, 1371

TMT_documants; TMT.PMO.MGT.0\%.009, Editors: Dawson, S., Roberts, S.

TMT_documants; TMT.PSC.TEC.07.003.REL01, Editors: Silva, D., Hickson, P., Steidel, C., Bolte, M.

TMT_documants; TMT.PSC.DRD.05.001.CCR18, Editors: Nelson, J.

Tominaga, N., Morokuma, T., Blinnikov, S. I., et al., 2011, ApJS, 193, 20

Tout, C. A., Wickramasinghe, D.T., Lau, H.H-B. et al., 2011, MNRAS, 410, 2458

Valenti, S., Benetti, S., Cappellaro, E. et al., 2008, MNRAS, 383, 1485

van der Horst, A. J., et al. 2009, ApJ, 699, 1087

Vreeswijk, P. M., Ledoux, C., Raassen, A. J. J. et al., 2013, A\&A, 549, 22

Vreeswijk, P. M., Ledoux, C., Smette, A. et al., 2007, $A \mathscr{E} A$, 468, 83

Ledoux, C., Vreeswijk, P. M., Smette, A. et al., 2009, $A \mathscr{E} A$, 506, 661

Wainwright, C., Berger, E., \& Penprase, B. E., 2007, ApJ, 657, 367

Wang, L. \& Wheeler, J. C., ARA\&A, 46, 433

Whalen, D. J., Even, W., Lovekin, C. C. et al., 2013a, submitted to ApJ, arXiv:1302.0436

Whalen, D. J., Even, W., Frey, L. H. et al., 2013b, submitted to ApJ, arXiv:1211.4979

Whalen, D. J., Heger, H., Chen, K. J. et al., 2013c, submitted to ApJ, arXiv:1211.1815

Wiersema, K., Curran, P. A., Kruhler, T., et al. 2012, MNRAS, 426, 2

Wiersema, K., van der Horst, A. J., Levan, A. J. et al., 2012b, MNRAS, 421, 1942

Wijers, R. A. M. J. \& Galama, T. J., 1999, ApJ, 523, 177

Woosley, S.E., Bloom, J.S., 2006, ARA $6 A$, 47, 507

Woosley, S. \& Janka, T., 2005, NatPh, 1, 147

Van dyk, S. D., Li, W., Cenko, S. B. et al., 2012, ApJ, 756, 131

Yoon, S. C., Langer, N., 2005, A\& A, 443, 643

Yoon, S. C., Grafener, G., Vink, J. S., et al., 2012, $A \mathscr{E} A$, 544, L11

Zhang, B., 2007, ChJAEA, 7, 1, 1 